\title{
COMPOSIÇÃO MINERAL DO LEITE MATERNO DE BANCOS DE LEITE ${ }^{1}$
}

\author{
Marcelo A. MORGANO ${ }^{2, *}$, Lidiane A. SOUZA², Júlio M. NETO ${ }^{3}$, Patrícia H. C. RONDÓ ${ }^{3}$
}

\section{RESUMO}

O leite humano é a primeira fonte de nutrientes que uma criança necessita para seu crescimento nos primeiros meses de vida. Os teores de muitos elementos minerais podem variar devido a alguns fatores, como a genética, a nutrição materna, o período de lactação e também entre os grupos étnicos. O objetivo deste trabalho foi de validar o uso do método de digestão por via seca (cinzas) e a técnica de espectrometria de emissão (ICP OES) para a quantificação dos elementos minerais Ca, P, Na, K, Mg, Zn, Fe e Mn presentes em 151 amostras de leite materno procedentes de doadoras de bancos de leite humano de Marília, SP. Os valores obtidos para os elementos minerais $\left(\mathrm{mg} \mathrm{L}^{-1}\right)$ nas amostras de leite variaram entre: $\mathrm{Ca}=145$ a $478 ; \mathrm{P}=87$ a $293 ; \mathrm{Fe}=0,03$ a 1,13 ; $\mathrm{K}=271$ a 1159; $\mathrm{Mg}=14,8$ a 60,0; $\mathrm{Na}=54$ a 933 e $\mathrm{Zn}=0,38$ a 5,82. Para o $\mathrm{Mn}$, os resultados obtidos foram inferiores ao limite de quantificação do método $\left(0,02 \mathrm{mg} \mathrm{L}^{-1}\right)$. Os valores obtidos para precisão e exatidão do método (\%) foram, respectivamente: Ca=1 e 96; $\mathrm{P}=1$ e $98 ; \mathrm{Fe}=4$ e $83 ; \mathrm{K}=1$ e $99 ; \mathrm{Mg}=1$ e $97 ; \mathrm{Na}=1$ e $98 ; \mathrm{Zn}=8$ e $96 ; \mathrm{Mn}=8$ e 82 . De acordo com os resultados obtidos, foi verificado que o método apresentou precisão e exatidão satisfatórias para todos os elementos estudados.

Palavras-chave: leite materno, composição mineral, espectrometria de emissão.

\section{SUMMARY}

MINERAL COMPOSITION OF HUMAN BANK MILK. Human milk is the child's primary source of nutrients during its first months of life. Mineral element levels can vary according to several factors such as genetic, the mother nutritional status, lactation period and ethnicity. The objective of the present study was the validation of a dry-ashed digestion method and ICP OES technique which were used for quantification of mineral elements ( $\mathrm{Ca}, \mathrm{P}, \mathrm{Na}, \mathrm{K}, \mathrm{Mg}, \mathrm{Zn}$, Fe and $\mathrm{Mn}$ ) in 151 samples of human milk collected from mothers in the city of Marília, SP. Values $\left(\mathrm{mg} \mathrm{L}^{-1}\right)$ obtained ranged from $\mathrm{Ca}=145$ to $478 ; \mathrm{P}=87$ to $293 ; \mathrm{Fe}=0.03$ to $1.13 ; \mathrm{K}=271$ to 1159; $\mathrm{Mg}=14.8$ to 60.0; $\mathrm{Na}=54$ to $933 \mathrm{e} \mathrm{Zn}=0.38$ to 5.82 . Mn was below the limit of quantification $\left(0.02 \mathrm{mg} \mathrm{L}^{-1}\right)$ for all analyzed samples. Method precision and accuracy (\%) values were respectively: $\mathrm{Ca}=1$ and $96 ; \mathrm{P}=1$ and $98 ; \mathrm{Fe}=4$ and $83 ; \mathrm{K}=1$ and 99 ; $\mathrm{Mg}=1$ and 97; $\mathrm{Na}=1$ and $98 ; \mathrm{Zn}=8$ and $96 ; \mathrm{Mn}=8$ and 82 . Results indicate that precision and accuracy were satisfactory for all elements analyzed on the present study.

Keywords: human milk, mineral composition, emission spectrometry.

\section{1 - INTRODUÇÃO}

O leite é a primeira alimentação humana e fonte de nutrientes para as funções biológicas, sendo considerado o melhor alimento para crianças, por ter papel muito importante na proteção imunológica contra doenças infecciosas, na adequação nutricional e no desenvolvimento afetivo e psicológico [2, 7, 17, 24, 26].

A composição do leite humano, especialmente quanto à presença de micronutrientes, é muito variada $[4,6,9$, $13,19]$ e pode ser influenciada por diversos fatores como a individualidade genética, a nutrição materna e o período de lactação. Ocorrem, também, variações entre grupos étnicos e entre mulheres. Para uma mesma mulher, são registradas variações no decorrer da lactação, ao longo do dia e durante uma mesma mamada, havendo diferenças entre o leite da frente e o último a sair (anterior e posterior) com alterações na concentração dos macro e dos micronutrientes [10, 19, 20, 23].

Os minerais são importantes para o crescimento, o desenvolvimento e a manutenção da saúde dos tecidos corporais [1]. São classificados em macroelementos o sódio, potássio, cálcio, magnésio e fósforo, entre outros, e

${ }^{1}$ Recebido para publicação em 13/05/2005. Aceito para publicação em 10/10/2005 (001529).

${ }_{2}^{2}$ TTAL/Centro de Química de Alimentos. Av Brasil, 2880 - CEP: 13073-001 - CP 139, Campinas-SP.

${ }^{3}$ USP/Faculdade de Saúde Pública. Av. Dr. Arnaldo, 715 - CEP: $01246-$ 904, São Paulo - SP.

*A quem a correspondência deve ser enviada. microelementos ou elementos traços, o cobalto, cobre, iodo, flúor, molibdênio, selênio, cromo, ferro, zinco e outros. As necessidades do organismo para os macroelementos são relativamente elevadas, enquanto para os elementos traços, são baixas [21].

A necessidade de micronutrientes para o recém-nascido é maior do que em crianças e adultos devido ao rápido crescimento corporal e também ao alto nível de atividade dos caminhos metabólicos envolvidos no crescimento, atividade física e combate a infecções, dentre outros. $\mathrm{O}$ atendimento a essa demanda é feito pelo leite materno, até que chegue a época de desmame [3]. O aleitamento materno também está associado à menor incidência de câncer de mama e de alguns tipos de câncer de ovário [16].

A composição dos elementos minerais presentes no leite materno apresenta variabilidade na sua composição e isto torna a avaliação do estado nutricional de lactentes uma tarefa extremamente complicada [4]. Sabe-se que a composição do leite humano varia conforme o estágio da lactação e mesmo entre as nutrizes [27]. Metodologias que permitam avaliar as concentrações dos micronutrientes do leite humano são necessárias, uma vez que as deficiências de micronutrientes em nutrizes podem acarretar prejuízo no crescimento e desenvolvimento dos bebês, predispondo-os à desnutrição. Os teores de minerais variam significativamente de mãe para mãe, sendo o colostro o tipo de leite que apresenta níveis mais elevados, seguido pelo leite de transição e pelo leite maduro [19]. 
O trabalho teve como objetivos:

a) avaliar uma metodologia de digestão por via seca (cinzas) e espectrometria de emissão para a quantificação de elementos minerais ( $\mathrm{Ca}, \mathrm{P}, \mathrm{Na}, \mathrm{K}, \mathrm{Mg}, \mathrm{Zn}$, Fe e Mn) em leite materno procedentes de banco de leite na cidade de Marília no estado de São Paulo;

b) conhecer o perfil micronutricional destas amostras de bancos de leite humano;

c) avaliar se o consumo diário médio dos microelementos presentes no leite materno destas mães está adequado aos bebês quanto às recomendações de Ingestão Dietética de Referência.

\section{2 - MATERIAL E MÉTODOS}

\section{1 - Amostragem}

No período de 2003 a 2004, amostras de leite maduro (25 a 35 dias após o parto) foram coletadas de 151 doadoras de bancos de leite humano na cidade de Marília, São Paulo.

O protocolo de estudo foi analisado e aprovado pelo Comitê de Ética em Pesquisa da Faculdade de Saúde Pública da Universidade de São Paulo.

\section{2 - Reagentes}

- $\quad$ Ácido nítrico P.A. (Merck);

- $\quad$ Padrões de solução Merck 10000 mg L-1 em solução de $\mathrm{HNO}_{3} 5 \%$ (v/v) de K, Ca, Na e Mg; e padrão Tec Lab de $1000 \mathrm{mg} \mathrm{L}^{-1}$ de $\mathrm{P}$, Fe, Mn e Zn com certificado de análise e rastreado com padrões NIST.

\section{3 - Método analítico}

A preparação da amostra foi adaptada de DONANGELO (1989) [8]: pipetaram-se 3,00 mL de cada amostra com três repetições analíticas em cápsula de porcelana; em seguida, a amostra foi seca em chapa de aquecimento e posteriormente incinerada em forno mufla a $450^{\circ} \mathrm{C}$ por 4 horas até a formação de cinzas brancas. As cinzas foram dissolvidas em 2,5 mL de ácido nítrico concentrado e transferidas quantitativamente para balão volumétrico de $50 \mathrm{~mL}$, completando-se o volume com água bidestilada.

A curva analítica foi preparada pela diluição de solução (Tec Lab) $1000 \mathrm{mg} \mathrm{L}^{-1}$ em solução de ácido nítrico 5\% (v/v) para concentração final de 0,01 a $2,5 \mathrm{mg} \mathrm{L}^{-1}$ para os elementos $\mathrm{Fe}$, Mn e $\mathrm{Zn}$; de 0,2 a $50 \mathrm{mg} \mathrm{L}^{-1}$ para o $\mathrm{P}$; e padrão (Merck) de 1,0 a 252,0 $\mathrm{mg} \mathrm{L}^{-1}$ para o K; de 0,21 a $52 \mathrm{mg}$ $\mathrm{L}^{-1}$ para os elementos $\mathrm{Ca}, \mathrm{Mg}$ e $\mathrm{Na}$.

Para a quantificação dos minerais cálcio, fósforo, ferro, potássio, magnésio, manganês, sódio e zinco foi utilizado um espectrômetro de emissão ótica com plasma de argônio induzido (ICP OES). O equipamento utilizado foi um ICP OES simultâneo, com visão axial VISTA MPX, VARIAN
(Mulgrave, Austrália). Para a nebulização das soluções foi empregado um nebulizador concêntrico ("see spray") acoplado a uma câmara ciclônica. As condições ótimas para determinação multielementar foram estabelecidas para o elemento manganês, conforme recomendação do fabricante. Os parâmetros instrumentais utilizados foram: potência do plasma $1,0 \mathrm{Kw}$; gás refrigerante (Ar) $15 \mathrm{~L}$ $\min ^{-1}$; gás auxiliar (Ar) $1,5 \mathrm{~L} \mathrm{~min}^{-1}$; pressão do nebulizador $200 \mathrm{kPa}$; gerador de rádio freqüência $40 \mathrm{MHz}$; velocidade da bomba $15 \mathrm{rpm}$; tempo de estabilização $10 \mathrm{~s}$; tempo de leitura $10 \mathrm{~s}$.

Os comprimentos de onda (nm) usados foram: cálcio 317,933; magnésio 279,553; fósforo 178,28 ; ferro 259,94 ; manganês 293,931; potássio 766,491; zinco 206,20; sódio 589,592 e fósforo 213,618 .

\section{4 - Parâmetros de validação}

\subsection{1- Preparo da amostra}

Para a validação [22] da metodologia empregada, utilizou-se uma amostra de leite certificada Whole Milk Powder (NIST/NBS-8435). Pesou-se em cápsula limpa e seca $1,0000 \mathrm{~g}$ da amostra previamente seca em estufa a $85^{\circ} \mathrm{C}$ por $4 \mathrm{~h}$. Em seguida, a amostra foi seca em chapa de aquecimento e posteriormente incinerada em mufla com aumento gradativo de temperatura até $450^{\circ} \mathrm{C}$ por $4 \mathrm{~h}$, com formação de cinzas brancas. As cinzas foram dissolvidas em 1,25 mL de ácido nítrico concentrado e transferidas quantitativamente para balão volumétrico de $25 \mathrm{~mL}$, completando-se o volume com água bidestilada. Foram feitas 4 repetições analíticas com a amostra certificada. Efetuou-se o branco analítico com os reagentes em triplicata. Para a leitura no equipamento, diluiu-se a amostra certificada 1:10 mL para o Ca, K, Na, P e Mg e as condições de operação do ICP OES foram as mesmas, conforme descrito anteriormente.

\subsection{2 - Linearidade}

A linearidade corresponde à capacidade de o método fornecer resultados diretamente proporcionais à concentração da substância em exame, dentro de uma determinada faixa de aplicação [15]. O coeficiente de correlação $\mathrm{R}$ foi o parâmetro usado para avaliar a qualidade da curva obtida, pois quanto mais próximo de 1,0, menor a dispersão do conjunto de pontos experimentais e menor a incerteza dos coeficientes de regressão estimados. Para verificar se a equação de regressão é estatisticamente significativa, foram efetuados os testes de ajuste do modelo linear, validade da regressão, sua eficiência e sua eficiência máxima [11, 12].

Para uma avaliação estatística, foi usado o teste t-student no qual se calcula o valor de $t_{r}$ ( $t$ calculado) com n -2 graus de liberdade, comparando com o valor $t$ tabelado para o nível de confiança desejado. Se o valor de $t_{r}$ é maior que o valor de $t$ tabelado, a correlação é significativa com a probabilidade calculada. 


\subsection{3 - Precisão, exatidão e sensibilidade}

A precisão do método, que representa a dispersão de resultados independentes repetidos de uma mesma amostra, amostras semelhantes ou padrões sob condições definidas $[14,15]$, foi determinada pelo coeficiente de variação (\%) de quatro repetições analíticas da amostra de leite certificada Whole Milk Powder (NIST/NBS-8435)

A exatidão foi determinada pelo erro relativo (\%), comparando-se o valor médio obtido experimentalmente da amostra de leite certificada com o valor certificado.

Os parâmetros utilizados para avaliar a sensibilidade foram os limites de detecção e de quantificação. O limite de detecção (LD) do método e o limite de quantificação (LQ) foram determinados usando os valores de leitura de 10 brancos analíticos com adição de padrão de metal nas concentrações de: 0,005 $\mathrm{mg} \mathrm{L}^{-1}$ para Fe, $\mathrm{Zn}$ e $\mathrm{Mn}$; 0,505 $\mathrm{mg} \mathrm{L}^{-1}$ para K; $0,105 \mathrm{mg} \mathrm{L}^{-1}$ para Ca, Mg e Na e $0,1 \mathrm{mg} \mathrm{L}^{-1}$ para o .

\section{3 - RESULTADOS E DISCUSSÃo}

\section{1 - Análise do material de referência}

Com o objetivo de verificar a exatidão do método de digestão por via seca (cinzas) e a técnica de espectrometria de emissão para a quantificação dos elementos minerais $\mathrm{Ca}, \mathrm{P}, \mathrm{Na}, \mathrm{K}, \mathrm{Mg}, \mathrm{Zn}, \mathrm{Fe}$ e $\mathrm{Mn}$, analisou-se o material de referência Whole Milk Powder (NIST/NBS8435). Na Tabela 1 pode ser visualizada a comparação entre os resultados obtidos e os valores certificados. O critério adotado para avaliar se os resultados foram satisfatórios foi o de escore $Z$ e o valor limite adotado foi de $(-3,3)$, que corresponde a um limite de confiança próximo a 99,7\%. Pode-se observar (Tabela 1) que o método de digestão por via seca (cinzas) e a técnica de espectrometria de emissão (ICP OES) foram adequados para análise do material.

$\mathrm{Na}$ análise do material de referência certificado, observou-se ausência de interferentes nos comprimentos de onda usados para todos os elementos minerais que pudessem comprometer a utilização da metodologia. O método mostrou-se seletivo para todos os minerais estudados.

Os resultados obtidos na avaliação dos parâmetros da linearidade do método encontram-se na Tabela 2. A ANVISA recomenda um coeficiente de correlação igual a 0,99 e o INMETRO um valor acima de 0,90 [5, 14], portanto, observando a Tabela 2 , verifica-se que as curvas analíticas para todos os elementos minerais estudados são lineares.

Os resultados obtidos na avaliação da precisão, exatidão e sensibilidade do métodos estão expressos na Tabela 3. A exatidão do método obtida foi satisfatória para os todos os elementos estudados e a precisão do método foi menor que $8,5 \%$ para todos os elementos.

TABELA 1 - Comparação dos resultados $\left(\mathrm{mg} \mathrm{kg}^{-1}\right)$ obtidos experimentalmente usando o método de digestão por via seca e quantificação por ICP OES, com o valor certificado do material de referência Whole Milk Powder (NIST/NBS-8435)

\begin{tabular}{lcccc}
\hline Elementos & Valor certificado & Valor médio encontrado $(\mathbf{n})$ & Erro relativo (\%) & Escore $\mathbf{Z}$ \\
\hline $\mathrm{Ca}$ & $9220 \pm 490$ & $9573 \pm 74$ & 3,8 & 3,5 \\
$\mathrm{P}$ & $7800 \pm 490$ & $8075 \pm 90$ & 16,6 & 0,72 \\
$\mathrm{Fe}$ & $1,8 \pm 1,1$ & $2,1 \pm 0,1$ & 1,4 & 0,56 \\
$\mathrm{~K}$ & $13630 \pm 470$ & $13815 \pm 136$ & 2,6 & 0,39 \\
$\mathrm{Mg}$ & $814 \pm 76$ & $835 \pm 7$ & 17,6 & 0,28 \\
$\mathrm{Mn}$ & $0,17 \pm 0,05$ & $0,20 \pm 0,02$ & 2,4 & 0,60 \\
$\mathrm{Na}$ & $3560 \pm 400$ & $3647 \pm 32$ & 3,1 & 0,22 \\
$\mathrm{Zn}$ & $28 \pm 3,1$ & $30 \pm 0,4$ & 0,64 \\
\hline
\end{tabular}

(n) Média de 4 repetições analíticas

TABELA 2 - Valores obtidos para os coeficientes de correlação linear das curvas analíticas, $\mathrm{t}_{\mathrm{r}}\left(t\right.$ calculado) e $\mathrm{t}_{\text {(tab) }}(t$ tabelado) para os minerais $\mathrm{Ca}, \mathrm{K}, \mathrm{Mg}, \mathrm{Mn}, \mathrm{Zn}, \mathrm{P}, \mathrm{Fe}$ e $\mathrm{Na}$

\begin{tabular}{|c|c|c|c|c|}
\hline Elementos & Equação linear & Coeficiente de correlação linear (R) & $t_{r}$ & $t_{\text {(tab) }}$ \\
\hline $\mathrm{Ca}$ & $y=12530 x+4026,4$ & 0,9999 & 141,407 & 2,776 \\
\hline $\mathrm{P}$ & $y=336,34 x+76,702$ & 0,9998 & 99,98 & 2,776 \\
\hline K & $y=45850 x+35258$ & 0,9998 & 86,585 & 3,182 \\
\hline $\mathrm{Mn}$ & $y=205944 x+55274$ & 0,9997 & 81,625 & 2,776 \\
\hline $\mathrm{Zn}$ & $y=828,43 x+8,7606$ & 0,9997 & 81,625 & 2,776 \\
\hline $\mathrm{Na}$ & $y=336731 x+28900$ & 1,000 & 1 & 1 \\
\hline
\end{tabular}


TABELA 3 - Parâmetros de validação usando uma amostra de leite certificada Whole Milk Powder (NIST/NBS-8435) e 4 repetições analíticas

\begin{tabular}{|c|c|c|c|c|c|c|}
\hline Elemento & $\lambda(\mathrm{nm})$ & $\mathbf{R}^{2}$ & LD (mg. L-1) & LQ (mg. L-1) & Exatidão (\%) & Precisão (\%) \\
\hline $\mathrm{Ca}$ & 317,933 & 0,9996 & 0,012 & 0,040 & 96 & 1 \\
\hline$P$ & 213,618 & 0,9996 & 0,009 & 0,030 & 97 & 1 \\
\hline K & 766,491 & 0,9994 & 0,132 & 0,440 & 99 & 1 \\
\hline $\mathrm{Mg}$ & 279,553 & 0,9984 & 0,012 & 0,040 & 97 & 1 \\
\hline $\mathrm{Zn}$ & 206,200 & 0,9994 & 0,003 & 0,010 & 97 & 8 \\
\hline
\end{tabular}

$\lambda=$ comprimento de onda; $\mathrm{R}^{2}=$ coeficiente de correlação da reta analítica; $\mathrm{LD}=$ limite de detecção ( 3 vezes o desvio-padrão de 10 leituras de padrões analíticos); LQ=limite de quantificação ( 10 vezes o desvio-padrão de 10 leituras de padrões analíticos); precisão: determinada pelo coeficiente de variação e o número de pontos da curva analítica igual a 5

\section{2 - Análise das amostras}

Na Tabela 4 são apresentados os resultados obtidos nas amostras de leite materno. Para o $\mathrm{Mn}$, todos os resultados encontrados nas amostras de leite materno estavam abaixo $0,02 \mathrm{mg} \mathrm{L}^{-1}$, valor que corresponde a duas vezes o limite de quantificação do método $\left(0,01 \mathrm{mg} \mathrm{L}^{-1}\right)$.

Os níveis dos elementos minerais encontrados neste estudo estão próximos aos obtidos no trabalho de OLIVEIRA (2003) [19], onde foram quantificados os seguintes elementos minerais $\mathrm{Ca}, \mathrm{Fe}, \mathrm{P}, \mathrm{Na}, \mathrm{K}, \mathrm{Mg}$, Mn e $\mathrm{Zn}$ em leite materno de mães doadoras do banco de leite do Hospital das Clínicas da Universidade Federal de Minas Gerais (UFMG), em Belo Horizonte. No trabalho de DONANGELO et al. (1989) [8], amostras de leite materno de mães do Hospital das Clínicas da cidade do Rio de Janeiro forneceram resultados para ferro e zinco compatíveis com os encontrados neste trabalho $\left(0,25-0,80 \mathrm{mg} \mathrm{L}^{-1}\right.$ e 0,2 a 4,0 $\mathrm{mg} \mathrm{L}^{-1}$, respectivamente).

As maiores variações na composição mineral foram observadas para os elementos ferro e sódio, seguidas pelo elemento zinco. Os resultados médios obtidos em todas as amostras de leite materno para o teor destes elementos apresentaram um coeficiente de variação de $94,3 \%$ para o ferro, $72,2 \%$ para o sódio e $41,5 \%$ para o zinco. Os elementos minerais cálcio, fósforo, potássio e magnésio apresentaram as menores variações, ou seja, o coeficiente de variação para as 151 amostras ficou abaixo de $27 \%$. Estas variações nas concentrações dos elementos minerais nas amostras de leite materno foram, provavelmente, devido a associações desses elementos no leite e à influência de fatores demográficos, nutricionais, psicológicos e socioeconômicos, que se acredita serem moduladores da secreção de micronutrientes no leite humano. Entre estes fatores estão o consumo alimentar ou o estado nutricional da mãe, estatura materna e o peso da criança, idade da mãe e a paridade, área onde reside, duração da gestação, entre outros.

Variações na composição mineral do leite materno também foram observadas no trabalho de OLIVEIRA (2003) [19]. Neste estudo, 108 mães que deram à luz no Hospital das Clínicas da UFMG doaram amostras de leite materno, e foi constatado que as maiores variações dos elementos minerais ocorrem para o leite maduro, seguido do leite de transição e pelo colostro para os macroelementos. Para os elementos traços, a maior variação foi para a fase de leite maduro, seguida pelo colostro e depois pelo leite de transição. Em todos os casos, as variações nos teores dos elementos minerais são maiores que $100 \%$.

Os teores médios dos microelementos, estimados quanto à ingestão diária pelas crianças e sua porcentagem de adequação à Ingestão Dietética de Referência (RDI) estão apresentados na Tabela 5. O consumo diário estimado foi feito baseando-se numa ingestão de $750 \mathrm{~mL}$ de leite pela criança durante os seis primeiros meses de vida [18]. A ingestão de cálcio, ferro e zinco atingiu mais que $94 \%$ do valor recomendado (valor médio), sendo que o valor para o magnésio ficou próximo a $66 \%$. O fósforo foi o único elemento cuja ingestão excedeu a $100 \%$ dos valores recomendados de ingestão diária. Os resultados médios, com exceção do elemento magnésio, indicam que os teores dos elementos minerais encontrados no leite materno atendem às necessidades calculadas para bom crescimento e desenvolvimento das crianças. Porém, apesar de o teor médio de ferro estar muito próximo a $100 \%$ da RDI, isso é preocupante, pois o valor médio apresenta desvio padrão muito alto (coeficiente de variação de 94\%), o que significa

TABELA 4 - Média, desvio-padrão (DP), valores máximos $\left(\mathrm{V}_{\text {máx }}\right)$ e valores mínimos $\left(\mathrm{V}_{\text {min }}\right)$ em mg $\mathrm{L}^{-1}$, obtidos para os elementos minerais para as análises das 151 amostras de leite humano

\begin{tabular}{lccccccc}
\hline Estatística & $\mathbf{C a}$ & $\mathbf{P}$ & $\mathbf{F e}$ & $\mathbf{K}$ & $\mathbf{M g}$ & $\mathbf{N a}$ & $\mathbf{Z n}$ \\
\hline Média & 263,55 & 159,26 & 0,35 & 489,76 & 26,53 & 207,21 & 2,65 \\
DP & 64,73 & 33,63 & 0,33 & 132,20 & 6,84 & 149,62 & 1,10 \\
V $_{\text {máx }}$ & 478,5 & 293,4 & 1,13 & 1159,3 & 60,05 & 933,0 & 5,82 \\
V $_{\text {min }}$ & 144,9 & 87,1 & 0,03 & 271,4 & 14,83, & 54,1 & 0,38 \\
\hline
\end{tabular}


TABELA 5 - Teores médios (desvio padrão $\mathrm{n}=151$ ) dos microelementos minerais ingeridos/dia pelos recém-nascidos e percentual relativo à Ingestão Dietética de Referência (RDI) por dia para os bebês de 0 a 6 meses

\begin{tabular}{lccc}
\hline Minerais & $\begin{array}{c}\text { Teor médio de minerais }(\mathbf{m g} / 750 \mathbf{~ m L} \text { de leite) } \\
\text { (adequação à RDI) }\end{array}$ & $\begin{array}{c}\text { Porcentagem de } \\
\text { adequação à RDI }\end{array}$ & $\begin{array}{c}\text { RDI } \\
\text { (mg/dia) }\end{array}$ \\
\hline Cálcio & $197,7(8,5)$ & 94 & 210 \\
Fósforo & $119,4(25,2)$ & 119 & 100 \\
Magnésio & $19,9(5,1)$ & 66 & 30 \\
Potássio & $367,3(99,2)$ & --- & -- \\
Sódio & $155,4(112,2)$ & -- & -- \\
Ferro & $0,26(0,25)$ & 96 & 0,27 \\
Zinco & $1,99(0,82)$ & 99 & 2,0 \\
\hline
\end{tabular}

que o leite materno de algumas mães pode apresentar níveis deficientes em ferro para os recém-nascidos. Do total das 151 amostras analisadas, 40\% apresentam níveis menores que $0,26 \mathrm{mg}$ de ferro.

\section{4 - CONCLUSÕES}

O método de digestão por via seca e a técnica multielementar usando a espectrometria de emissão apresentaram níveis de precisão e exatidão satisfatórios para a quantificação dos elementos $\mathrm{Ca}, \mathrm{P}, \mathrm{Na}, \mathrm{K}, \mathrm{Mg}, \mathrm{Zn}, \mathrm{Fe}$ e $\mathrm{Mn}$ em amostras de leite materno.

Os resultados médios obtidos dos teores dos elementos minerais, com exceção do magnésio, presentes nas amostras de leite materno das mães da cidade de Marília, indicam que os valores atendem à porcentagem de adequação à Ingestão Dietética de Referência para os recém-nascidos de 0 a 6 meses.

O teor médio do elemento mineral ferro foi o que apresentou a maior variação entre as amostras de leite materno, seguido pelo sódio e zinco.

\section{5 - REFERÊNCIAS BIBLIOGRÁFICAS}

[1] AL-AWADI, F.M.; SRIKUMAR, T.S. Trace-element status in milk and plasma of Kuwaiti and non-Kuwaiti lactating mothers. Nutrition, v. 6, n. 11-12, p. 1069-1073, 2000.

[2] American Academy of Pediatrics. Breast-feeding, a commentary in celebration of the International Year of the Child 1979. Pediatrics, v. 62, p. 591-601, 1978.

[3] BATES, C.J.; PRENTICE A. Breast milk as a source of vitamins, essential minerals and trace elements. Pharmacol. Ther., v. 62, p. 193-220, 1994.

[4] BENEMARIYA, H.; ROBBERECHT, H.; DEELSTRA, H. Cooper, zinc and selenium concentration in milk from middle-class women in Burundi (Africa) throughout the first 10 months of lactation. Sci. Total. Environ., v. 164, p. 161-174, 1995.

[5] BRASIL. Ministério da Saúde. Secretaria de Vigilância Sanitária. Resolução RE no 899, de 29 de maio de 2003. Determina a publicação do Guia para validação de métodos analíticos e bioanalíticos (on-line). Disponível em: < http://elegis.bvs.br/leisref/public/showAct. php?id $=15132 \&$ word $=$ valida $\%$ c3\%a7ao $>$. Acesso em: 02 de maio de 2005.
[6] CONI, E.; STACCHINI, A.; CAROLI, S.; FALCONIERI, P. Analytical approach to obtaining reference values for minor and trace elements in human milk. J. Anal. At. Spectrom., v. 5, p. 581-586, 1990.

[7] DEWEY, K.G.; HEINING, M.J.; NOMMSEN, L.A.; LÖNNERDAL, B, 1990. Growth patterns of breastfed infants during the first year of life: The darling study. In: ATKINSON, S.A. et al. Breast-feeding, Nutrition, Infection and Infant Growth in Developed and Emerging Countries. St. John's Newfoundland: ARTS Biomedical Publishers and Distributors; 1990. p. 269-282.

[8] DONANGELO, C.M.; TRUGO, M.F.; KOURY, J.C.; BARRETO, S.M.I.; FREITAS, L.A.; FELDHEIM, W. Iron, zinc, folate and vitamin B12 nutritional status and milk composition of low-income Brazilian mothers. Eur. J. Clin. Nutr., v. 43, p. 253-266, 1989.

[9] DOREA, J.G. Iron and copper in human milk. Nutrition, v. 16, p. 209-220, 2000.

[10] EMMETT, P.M.; ROGERS, I.S. Properties of human milk and their relationship with maternal nutrition. Early Hum. Dev., v. 49, p. 7-28, 1997.

[11] Eurachem Working Group. The Fitness for Purpose of Analytical Methods, a Laboratory Guide to Method Validation and Related Topics, 1998. p. 121.

[12] European Commission; Guidance Document on Residue Analytical Methods, SANCO/825/00, 2000. p. 85.

[13] GROSS, R.; HÄNSEL, H.; SCHULTINK, W.; SHIRIMPTON, R.; MATULESSI, R.; GROSS, G. Moderate zinc and vitamin A deficiency in breast milk of mothers from East-Jakarta. Eur. J. Clin. Nutr., v. 52, p. 884-890, 1998.

[14] Instituto Nacional de Metrologia, Normalização e Qualidade Industrial (INMETRO); Orientação sobre Validação de Métodos de Ensaios Químicos, DOQCGCRE-008, 2003. p. 32.

[15] International Conference on Harmonization (ICH); Validation of Analytical Procedures: Methodology, Q2B (CPMP/ICH/281/95), 1995.

[16] LANG, S. Aleitamento do Lactente. São Paulo: Santos, 1999. 179 p.

[17] MONTEIRO, C.; REA, M.F.; VICTORA, C.G. Can infant mortality be reduced by promoting breast-feeding? Evidence from São Paulo city. Health Policy Plan., v. 5, p. 23-29, 1990.

[18] National Research Council. Recommended Dietary Allowances. $10^{\text {th }}$ ed. Washington: National Academy 
Press, 1989. 285 p.

[19] OLIVEIRA, M.C.C. Práticas de Amamentação, Teores de Minerais e Vitamina A no Leite Humano em Diferentes Fases de Lactação segundo Variáveis Maternas. Belo Horizonte, 2003. 60 p. Tese (Mestrado). Faculdade de Farmácia/Universidade Federal de Minas Gerais (UFMG).

[20] PICCIANO, M.F. Nutrient composition of human milk. Pediatr. Clin. North Am., v. 48, n. 1, p. 53-67, 2001.

[21] QUEIROZ, S.S. O papel do ferro na alimentação infantil. In: Departamento de Nutrição da Sociedade Brasileira de Pediatria. Temas de Nutrição em Pediatria. [s.i.]: Nestlé, 2001a. p. 8-9.

[22] RIBANI, M.; BOTTOLI, C.B.G.; COLLINS, C.H., JARDIM, I.C.S.F.; MELO, L.F.C. Validação em métodos cromatográficos e eletroforéticos. Quím. Nova, v. 27, p. 771-780, 2004.

[23] VALDÉS, V.; SÁNCHEZ, A.P.; LABBOCK, M. Manejo Clínico da Lactação. Assistência à Nutriz e ao
Lactente. Rio de Janeiro: Revinter, 1996. 128 p.

[24] VICTORA C.G. Infection and disease: the impact of early weaning. Food Nutr. Bull., v. 17, p. 390-396, 1996.

[25] VICTORA, C.G.; SMITH, P.G.; VAUGHAN, J.P.; NOBRE, L.C.; TEIXEIRA, A.M.B.; FUCHS, S.M.C. Evidence for protection by breast-feeding against infant deaths from infections diseases in Brazil. Lancet, v. 2, p. 319-321, 1987.

[26] VICTORA, C.G. The association between wasting and studying: an international perspective. J. Nutr., v. 122, p. 1105-1110, 1992.

[27] YOSHINAGA, J.; LI, J.Z.; SUZUKI, T.; KARITA, K.; ABE, M.; FUJI, H. Trace elements in human transitory milk. Biol. Trace Elem. Res., v. 31, p. 159-170, 1991.

\section{6 - AGRADECIMENTOS}

Os autores agradecem ao suporte financeiro da FAPESP (Processo no 2003/04061-2). 